\title{
A política de saúde portuguesa na austeridade: um olhar do Serviço Social
}

\author{
Jorge Lopes da Costa \\ Universidade de Lisboa (ISCSP) - Portugal
}

Maria Irene Carvalho

Universidade de Lisboa (ISCSP) - Portugal

Este artigo mantém a ortografia vigente em Portugal.

\author{
Carla Pinto \\ Universidade de Lisboa (ISCSP) - Portugal
}

\section{A política de saúde portuguesa na austeridade: um olhar do Serviço Social}

Resumo: Este artigo analisa a política de saúde em Portugal na perspetiva do Serviço Social em tempo de austeridade. Identificamos as competências e atribuições do Serviço Social na saúde e evidenciamos o trajeto do Serviço Nacional de Saúde, desde a sua génese até aos seus últimos desenvolvimentos. Salienta-se as alterações na política de saúde que ocorreram durante a crise económica-financeira do Estado entre 2010 e 2015 e reflecte-se sobre o impacto dessas mudanças no Serviço Social considerando, simultaneamente, cenários onde a escassez de recursos e o recurso à privatização do setor são uma realidade. Para atingir os objetivos a que nos propusemos optámos por uma metodologia centrada na análise documental e assente na experiência dos autores-evidence-based practice.

Palavras-chave: Serviço Social. Políticas de saúde. Crise financeira. Assistentes sociais da saúde.

\section{The Portuguese health policy in austerity: the perspective of Social Work}

Abstract: This paper analyzes Portugal's health policy from the perspective of Social Work in time of austerity. We have identified the competencies and responsibilities of Social Work in the field of health and evidenced the trajectory of the National Health Service since its inception until its recent developments. Notably, changes occurred to the health policy during the economic crisis of the State between 2010 and 2015, and an analysis was made of the impact of these changes in Social Service, while considering scenarios where the scarcity of resources and privatization of the sector are both common facts. In order to achieve the goals we have set, we opted for an approach focused on documentary analysis, based on the experience of the authors (evidence-based practice).

Keywords: Social Work. Health policies. Financial crisis. Health social workers. 


\section{Introdução}

O Serviço Social tem uma relação próxima com a saúde mesmo antes da existência dos modernos sistemas de saúde (de base bismarckiana, seguros; e de base beveridgiana, serviço nacional de saúde). Durante décadas, ligada a um compromisso de caridade, prestou-se assistência sanitária aos doentes com carência económica por instituições públicas repressivas e por benfeitores privados. Os profissionais de Serviço Social inseridos nestas dinâmicas processuais desenvolviam funções direcionadas para a compreensão das condições psíquicas e sociais que estavam na origem da doença, que impediam ou dificultavam o tratamento dos doentes (MARTINS, 1995).

Em Portugal, antes da criação do Serviço Nacional de Saúde (SNS), as primeiras alusões ao Serviço Social constam da Lei n. 2011/1946, de 2 de Abril (PORTUGAL, 1946), na qual se elencam as especificidades relativamente à alocação de recursos humanos e à formação de assistentes sociais no quadro dos hospitais regionais e sub-regionais. Apesar dessa relação histórica com a saúde foi apenas com a criação do SNS em 1979 (PORTUGAL, 1979) que em Portugal os assistentes sociais passaram a integrar o recém-criado SNS. Os profissionais inseriram-se: em hospitais (centrais, gerais ou especializados); em centros de saúde e noutras áreas de intervenção preventiva ou terapêutica. Atualmente a categoria profissional integra-se nas organizações e entidades públicas prestadoras de cuidados de saúde, designadamente em agrupamentos de centros de saúde, estabelecimentos hospitalares e unidades locais de saúde (PORTUGAL, 2011). Os Agrupamentos de Centros de Saúde (ACES) integram Unidades de Saúde Familiar (USF), enquanto os hospitais estão agregados a Centros Hospitalares $(\mathrm{CH})$ e articulados com as unidades de saúde local que visam aumentar a coordenação da prestação entre níveis de cuidados.

$\mathrm{Na}$ área da continuidade de cuidados o sistema de saúde une-se à Segurança Social para satisfazer, em simultâneo, necessidades de cariz social e de apoio clínico numa abordagem Welfare Mix (EVERS; WINTERSBERGER, 1990). Esta visão integrada da governação da saúde, em que participam vários setores da sociedade e que prevê um compromisso alargado (SAKELLARIDES, 2010) acabou por viabilizar numa primeira fase, a criação da Rede Nacional de Cuidados Continuados Integrados (RNCCI, 2006) e a sua extensão recente aos cuidados integrados de saúde mental em 2015, e numa segunda fase, à conceção de uma rede de cuidados paliativos em 2012.

Os profissionais de Serviço Social têm competências profissionais atribuídas na organização do sistema de saúde. Estas estão tipificadas em legislação específica e incluem, de forma genérica, as atribuições que vigoram atualmente nos serviços e estabelecimentos dependentes do Ministério da Saúde. As suas principais funções são:

- Identificar e analisar os problemas e as necessidades de apoio dos utentes, elaborando o respetivo diagnóstico social;

- Proceder ao acompanhamento e apoio psicossocial dos utentes e respetivas famílias, no quadro dos grupos sociais em que se integram, mediante a elaboração prévia de planos de intervenção social;

- Participar na definição, promoção e concretização das políticas de intervenção social a cargo dos respetivos serviços ou estabelecimentos;

- Definir, executar e avaliar programas e projetos de intervenção comunitária na área de influência;

- Analisar, selecionar, elaborar e registar a informação no âmbito da intervenção profissional e da investigação científico-profissional;

- Assegurar a continuidade dos cuidados sociais a prestar, em articulação com os parceiros da comunidade;

- Envolver e orientar utentes, famílias e grupos de autoconhecimento na procura de cuidados adequados às suas necessidades;

- Articular-se com os restantes profissionais dos serviços ou estabelecimento para melhor garantir qualidade, humanização e eficiência na prestação de cuidados;

- Relatar, informar e acompanhar, sempre que necessário e de forma sistemática, situações sociais problemáticas, em especial as relacionadas com crianças/jovens, idosos, doentes e vítimas de crimes ou de exclusão (PORTUGAL, 2002).

O Departamento de Modernização e Recursos de Saúde estabeleceu este conjunto de atribuições acima referidas para serem exercidas em meio hospitalar e no contexto dos cuidados de saúde primários e também previu que os assistentes sociais devem reportar superiormente ao órgão dirigente máximo (PORTUGAL, 2002).

Aquando da criação da RNCCI foram identificadas novas atribuições que estão no alinhamento da praxis na transição dos níveis de cuidados. Estas ficaram expressas num Manual de Boas Práticas para Assistentes Sociais (DGS, 2007), sob a coordenação e responsabilidade da Direção Geral da Saúde. Assim, confirma-se que o papel do assistente social na transição dos cuidados considera as seguintes dimensões: 


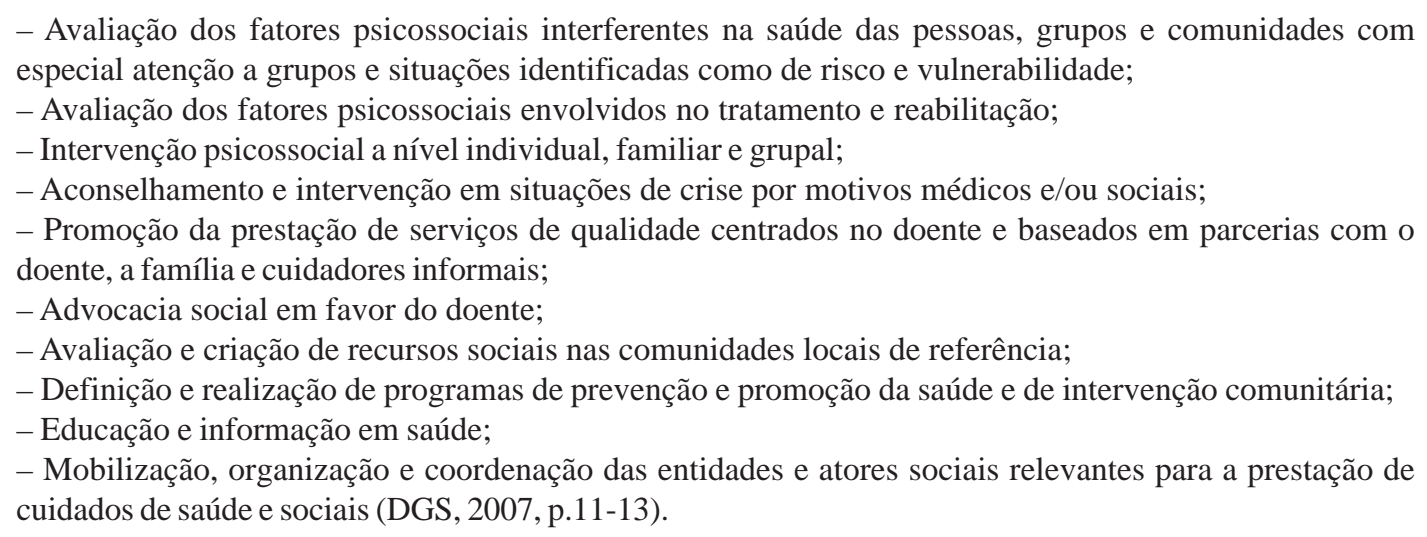

O Serviço Social na saúde tem a incumbência de adequar a perspetiva conciliadora da profissão, que lhe é conferida pela essência da relação de ajuda às necessidades dos doentes e suas famílias. Tem também a responsabilidade de defender os direitos dos cidadãos quando se colocam em causa o acesso, a adequação e a eficácia dos cuidados preventivos, curativos e de reabilitação. $\mathrm{O}$ assistente social assume as dimensões de intervenção social na área da saúde e nesse campo de ação, através dos instrumentos que dispõe e das suas habilidades (saber-fazer), fundamenta a prática num quadro de referência estruturado teoricamente (saber teórico). Este exercício reconhece os utentes como os protagonistas dos seus projetos de vida, apoia a defesa dos seus interesses e ajuda-os a desenvolver a perceção sobre as suas condições de vida e sobre a realidade que os envolve (CAMPOS, 2003; VISCARRET, 2007).

A abordagem do Serviço Social apesar de ser compatível com uma tipologia de atuação profissional que é entendida como promocional (por oposição à assistencialista), alicerça-se na participação/motivação e na orientação da ação para a promoção da saúde dos indivíduos (RESTREPO, 2003). Contudo, vai mais além e inclui-se no domínio das práticas sociais contemporâneas onde se assume a preocupação com a interação das pessoas no seu meio envolvente. Há maior plasticidade e abertura e a sua aplicação pode ser atravessada, tanto por dimensões individuais como coletivas, num dinamismo que reconfigura o Serviço Social do século 21 num modelo de convergência, no qual a concertação, o diálogo e o trabalho pluridisciplinar e em rede são peças fundamentais.

Por outro lado, o Serviço Social também assegura um papel crucial na construção e no desenho das políticas, o que lhe permite discutir medidas mais justas e adequadas pela experiência aferida do exercício profissional

o Serviço Social também assegura um papel crucial na construção e no desenho das políticas, o que lhe permite discutir medidas mais justas e adequadas pela experiência aferida do exercício profissional e da avaliação da sua intervenção... e da avaliação da sua intervenção (BECKET, 2009). O assistente social nas políticas de saúde e em particular no SNS caracteriza-se por atuar na área do planeamento da gestão das medidas políticas, mas também por apoiar a sua execução. Entenda-se gestão social das políticas como a capacidade para conjugar a procura e as necessidades dos cidadãos, considerando os instrumentos disponíveis para a população: programas, projetos e instituições vigentes. Neste domínio, encontra-se apto para contribuir com o seu conhecimento para formular ou redesenhar sistemas de proteção social, especialmente, para as pessoas que vivem em situação de maior vulnerabilidade. No papel de executor de políticas para além de participar no seu processo de inclusão também é chamado a advertir e a interpretar os critérios que validam a sua admissibilidade. Nessa medida, a sua atuação confronta-se frequentemente com a desarmonia que existe entre o acesso ao direito à saúde, aos fatores dessa elegibilidade e às necessidades objetivas dos cidadãos. Também se consideram as exigências formais que funcionam como critérios de seleção (área de residência, patologia) e o cumprimento de regras/ metas que estão ligadas a determinadas fontes de financiamento das medidas políticas.

O enviesamento gerado entre a legislação social existente e os recursos disponíveis para a população é evidente na atividade do assistente social, sendo da sua responsabilidade ir ao encontro de respostas alternativas que visem a defesa dos direitos e o acesso a serviços de saúde. Um olhar sobre a política de saúde 
portuguesa demonstra a forma como o Serviço Social contribui para a efetividade do direito universal à saúde e a serviços de qualidade reconhecendo o peso das Determinantes Sociais da Saúde (DSS) (DAHLGREN; WHITEHEAD, 2006). Estas determinantes acabam por definir as circunstâncias do contexto político, social e cultural e implicam o conhecimento da realidade social dos cidadãos, permitindo o desenho de intervenções conducentes à melhoria das suas condições de saúde (CRAIG; BEJAN; MUSKAT, 2013). Cabe ainda acrescentar que esta perspetiva das DSS tem enquadramento nos valores fundamentais do Serviço Social, salientando o facto da Federação Internacional dos Assistentes Sociais (FIAS) defender a saúde, como uma matéria simultaneamente dos direitos humanos e da justiça social (KARBAN, 2010).

\section{O Serviço Nacional de Saúde em Portugal}

O SNS foi criado em 1979, e erigido numa base democrática, assente nos princípios da universalidade, generalidade e gratuitidade. Ficou consagrado por regulação jurídica, que o Estado passaria a assegurar o direito à saúde a todos os cidadãos (PORTUGAL, 1979) de acordo com a Constituição Portuguesa de 1976 (art. n. 64), que afirmava esse direito e o dever de o defender e promover. Na realidade, apesar da salvaguarda jurídica, o desenho do SNS levaria cerca de uma década a capitalizar-se, fruto de constrangimentos e lutas internas entre Estado e o corporativismo médico (MOZZICAFREDDO, 2000).

Campos e Simões (2011) ilustram em forma de síntese a evolução do SNS em Portugal. Entre 1970 a 1982 foi o período de criação e expansão do SNS; entre 1982 a 1995, assistiu-se à sua implementação e desenvolvimento onde a agenda predominante é a mudança da fronteira entre público e privado. Este período, também cunhado pela adesão de Portugal à Comunidade Económica Europeia (CEE), em 1986, proporcionou uma maior abertura do país ao exterior, até então inexistente, e iniciou uma mudança clara no rumo da saúde, nomeadamente, no aumento do número de unidades hospitalares e na procura da população por serviços de urgência.

Entre 1995 a 2002, predomina a ideia de aumento dos serviços existentes em que é visível o recuo na narrativa da privatização do SNS. Este intervalo cronológico refere-se a um novo impulso do SNS, que propõe uma reforma que possa inovar o sistema de saúde, conferindo-lhe maior elasticidade e autonomia, pela via de novos modelos de organização e cuidados de saúde. É também coincidente com a primeira estratégia em saúde, entre o ano de 1998 e de 2002, e com as 27 áreas de ação estabelecidas em metas tangíveis a 5 e a 10 anos, extensíveis às cinco regiões administrativas que subdividem a prestação de serviços de saúde a nível nacional.

Entre 2002 a 2005, assistimos a uma combinação de estratégias centradas na eficiência do sistema com um discurso de mudança para um sistema misto entre público e privado e de substituição do Serviço Nacional de Saúde por um Sistema Nacional de Saúde. Esta fase corresponde a um determinado ciclo político, e fica conhecida pelos significativos contributos legislativos que alargaram o espectro da prestação de cuidados ao sector privado e ao terceiro sector. Esta rede de cuidados de saúde, apoiada na complementaridade sectorial e financiada pelo estado, conduziu a novas políticas assentes, por exemplo, no novo regime jurídico da gestão hospitalar que previu a subcontratação de entidades privadas para o sector público, a criação de unidades de natureza de sociedades anónimas, a gestão privada de hospitais públicos através de Parcerias Público-privadas, e a introdução do contrato individual de trabalho como regime aplicável aos trabalhadores do SNS.

Entre 2005 a 2009 há um reencontro com políticas que acentuam a manutenção da referência ideológica do SNS e alargamento e modernização de serviços ao mesmo tempo em que se mantêm e acentuam as estratégias que visam os ganhos de eficiência. Uma das medidas, ao nível dos cuidados de saúde primários, promovida pelo XVII Governo Constitucional, traduziu-se no reconhecimento deste nível de cuidados como estratégia fundamental. Neste âmbito, criaram-se Unidades de Saúde Familiar (USF), com distintos níveis de desempenho e de complexidade, com o intuito de centralizar os cuidados numa lógica de proximidade, principalmente a aproximação da medicina geral e familiar aos utentes. A jusante, e considerando o início de um novo nível de cuidados, foi também criada a Rede Nacional de Cuidados Continuados Integrados (Lei 101/2006, de 6 de Junho) - RNCCI.

Desde 2010 que o Memorando de Entendimento se fez sentir no sistema de saúde português, com a aplicação de medidas de austeridade impostas pelas revisões do Programa de Estabilidade e Crescimento e pelas obrigações do estado português no âmbito do Plano de Assistência Financeira. Nesse sentido, foi necessário empreender reformas que pudessem oferecer sustentabilidade, e ao mesmo tempo uma maior cobertura da prestação de serviços à população portuguesa, tanto na área do financiamento, como na do medicamento e da monitorização da prescrição, ou ainda no sector farmacêutico e no sistema de compras e de aprovisionamento de material médico, conforme o Relatório da Primavera do Observatório Português dos Sistemas de Saúde - OPSS (2011). Ao nível dos Cuidados de Saúde Primários, as opções seguiram a linha de reforço das Unidades de Saúde Familiares (USF) e a garantia da presença de médicos de família de forma mais ampla, 
pelo território nacional. No que se refere aos cuidados hospitalares, importou ressarcir os fornecedores das dívidas adquiridas, através de um plano concertado, com medidas que reduzissem custos operacionais, investir na área da qualidade, ao nível dos processos, das normas e dos sistemas de auditoria, bem como consolidar a reforma da rede hospitalar, reorganizando e racionalizando recursos humanos e serviços de ambulatório. Finalmente, em algumas áreas transversais importou investir na criação de um sistema eletrónico para o registo médico, e tentar reduzir custos relacionados com o transporte de doentes (OPSS, 2011, p. 80).

Atualmente são ainda utilizadas estratégias que passam pela separação entre os papéis do estado enquanto financiador, regulador e prestador de cuidados de saúde; pelo estímulo à concorrência entre setor público e privado e pela remercadorização (SILVA, 2012). No Quadro 1 apresentam-se importantes instrumentos de reforma no Sistema de Saúde.

\section{Quadro 1 - Instrumentos de reforma em Portugal - 2012}

\begin{tabular}{|l|l|}
\hline $\begin{array}{l}\text { Separação entre financiamento e prestação dos } \\
\text { cuidados }\end{array}$ & $\begin{array}{l}\text { Contratualização entre o Estado e hospitais públicos } \\
\text { Gestão por objetivos }\end{array}$ \\
\hline $\begin{array}{l}\text { Competição entre hospitais públicos e entre hos- } \\
\text { pitais públicos e privados - mercado interno }\end{array}$ & $\begin{array}{l}\text { Hospitais privados incluídos na rede pública } \\
\text { Deduções fiscais } \\
\text { Sistema remuneratório }\end{array}$ \\
\hline $\begin{array}{l}\text { Diminuição do papel do estado enquanto } \\
\text { prestador }\end{array}$ & $\begin{array}{l}\text { Acordo de cirurgia concessionada } \\
\text { Alargamento aos privados de novas áreas de prestação de cuidados (como } \\
\text { os cuidados continuados, medicina oral com o cheque dentista) }\end{array}$ \\
\hline Parcerias público privadas
\end{tabular}

Fonte: Silva(2012).

Neste espaço-tempo assiste-se, portanto, a uma maior racionalidade (controlo) no acesso aos serviços de saúde, designadamente ao aumento dos tempos de espera para exames e consultas; à subida das taxas moderadoras (consultas e exames); à redução das isenções para medicamentos e serviços de transporte, ao controlo no acesso a ajudas técnicas e a produtos de apoio e à redução de programas de promoção da saúde, bem como à estagnação da Rede Nacional de Cuidados Continuados Integrados (RNCCI).

\section{As políticas de saúde no plano de assistência financeira}

Quando a crise económica e financeira se instalou entre 2010/2015, e essa, se traduziu num austero e controverso aumento de impostos e de deduções salariais, o número de problemas de ordem física e mental e as suas manifestações na saúde dos mais frágeis aumentou consideravelmente (FALAGAS, 2009; MARMOT, 2009; OPSS, 2011). Instâncias como o Eurostat e o Banco de Portugal aconselharam Portugal para a criação de estratégias nacionais, dadas as lacunas estruturais na prestação de cuidados de saúde, por entidades e agentes de relevo. Para estas entidades ficavam explícitos os problemas estruturantes, aos quais se deveria dar resposta: insustentabilidade financeira, carência de planeamento estratégico e elevado nível de ineficiência (DELOITTE PORTUGAL, 2011; OPSS, 2011). Na verdade, todas as linhas de orientação para a governação na saúde e a própria agenda nacional, ficaram subjugadas a uma estratégia global, que condicionou metas e escolhas propostas pelo governo em funções, designadamente, pelo que dependia do Orçamento para a saúde que sofria, progressivamente, uma redução na despesa do estado com a função social Saúde (PORTUGAL, 2012).

O Memorando de Entendimento fez-se sentir no sistema de saúde português e foi necessário empreender reformas que pudessem oferecer sustentabilidade e garantir a cobertura da prestação de serviços de qualidade. As circunstâncias obrigaram a racionalizar medidas e a selecionar prioridades de atuação. A estratégia atravessou áreas como o financiamento, a política do medicamento, o setor farmacêutico e o sistema de 
compras e de aprovisionamento de material médico (OPSS, 2011). Uma das linhas de atuação traduziu-se pelo aumento das taxas moderadoras através de novos cálculos indexados à inflação e associados à capacidade para se fazer face ao seu pagamento, confirmando um claro retrocesso na definição do conceito do SNS.

Neste plano, os assistentes sociais foram desafiados a promover a justiça social e a contender a opressão, a desigualdade e a pobreza (INTERNATIONAL FEDERATION OF SOCIAL WORKERS, 2012). A crise que inicialmente afetou o setor financeiro, cedo se transformou numa crise social com implicações severas para os mais desprotegidos, mercê do recuo do investimento em políticas públicas. Neste período, cabia ao sistema de proteção social envidar esforços e mobilizar recursos para assumir a destabilização vivida pelos vários setores da atividade produtiva. Porém, a reação governamental não foi suficiente porque visava especialmente a redução dos défices orçamentais (LUNDY, 2011).

Nesta medida, as alterações provocadas pela instabilidade do fenómeno conseguiram afetar direitos sociais há muito conquistados, como: “o direito a um padrão de vida adequado ao nível da saúde e bem-estar (...), tratamento médico e os serviços sociais tais como a proteção no caso de desemprego, invalidez, viuvez e velhice" (UNITED NATIONS, 1948, art. 25). As repercussões da estratégia utilizada por Portugal, também partilhada por outros países europeus (Espanha, Itália, Grécia) acabaram por criar dificuldades e reduzir a autonomia de decisão pelo receio de uma perda efetiva de confiança nos mercados financeiros (IFE, 2012).

\section{Mudanças na política de saúde}

A eficiência na gestão dos recursos da saúde resultou mais pela imposição externa do cumprimento do programa de ajustamento do que propriamente pela estratégia política que visa ganhos em saúde (OPSS, 2014). Os efeitos da contenção de recursos serviram para o célere pagamento de uma boa parte da dívida acumulada pelo SNS a parceiros e a entidades credoras, mas o facto de se terem equilibrado as obrigações, deixou de lado uma importante base de sustentação nos projetos de médio e longo prazo dos serviços e dos diferentes níveis de cuidados disponíveis para a população portuguesa. No quadro das ineficiências geradas na governação da saúde da última década destacam-se algumas áreas de relevo.

$\mathrm{Na}$ área dos Cuidados de saúde primários, CSP, a inexistência de um modelo de financiamento explícito e a ausência de uma política de investimento em recursos humanos condicionaram a capacidade operativa e a credibilidade do processo de reforma (OPSS, 2010). Os sistemas de informação existentes estiveram aquém do desejável, bem como se detetaram fragilidades em unidades funcionais (OPSS, 2014).

Na área do medicamento verificou-se, quer por influência dos agentes económicos ou por ausência de monitorização, uma utilização indevida de antibióticos (cefalosporinas e quinolonas) que não é compatível nem com padrões de boa prática médica, e menos ainda, com o que se entende por práticas que visam à racionalidade económica (PALMA, 2002).

$\mathrm{Na}$ área das Parcerias Público Privadas (PPP) houve imprudência no que se refere ao interesse público dos contratos celebrados dada a inexistência de projetos-piloto ou experiências internacionais reconhecidas empiricamente nas áreas em questão. Também se acrescenta a displicência com que processos e instrumentos de gestão foram adotados sem uma base de disciplina devidamente fundamentada e documentada (OPSS, 2009).

$\mathrm{Na}$ área da saúde mental a política de desinstitucionalização (fecho de unidades hospitalares psiquiátricas e a aposta no apoio de proximidade) ficou carecida de um importante investimento para se chegar a uma política de base comunitária. A este domínio podem aduzir-se os dados que se referem ao aumento da taxa de incidência de depressão e à expressão do consumo de álcool e de canabinóides na população jovem (OPSS, 2014).

Em matéria de eficiência processual e de melhoria de qualidade da prestação dos serviços de saúde pode fazer-se referência à aposta nos novos processos de contratualização de desempenho nas Unidades Hospitalares e nos Cuidados de Saúde Primários (CSP). No âmbito dos CSP estabeleceram-se instrumentos de incentivos como o regime remuneratório e lançou-se um novo modelo organizacional para o conjunto de CSP, os Agrupamentos de Centros de Saúde (ACES). Na realidade, houve também a abertura de vagas para o internato de Medicina Geral e Familiar e criaram-se novas Unidades de Saúde Familiar (USF). Nos cuidados diferenciados ficou patente a melhoria da performance da espera média para cirurgia (OPSS, 2009) e no que se refere a consultas externas, foi implementada uma nova legislação que visa o cumprimento de "tempos mínimos garantidos" de atendimento.

Quanto a ganhos para a saúde na vertente salutogénica ou de promoção da saúde (ANTONOVSKY, 1991) assinalaram-se importantes marcos, para os quais contribuíram a nova lei do tabaco (DGS, 2011), a alteração relativa à interrupção voluntária da gravidez e o investimento na criação de uma plataforma contra a obesidade (CARMO et al., 2008). De referir também estão os programas relacionados com as alterações dos estilos de vida: o Programa Nacional para a Promoção da Alimentação Saudável e o Regime de Fruta Escolar (OPSS, 2014). 
$\mathrm{Na}$ vertente patogénica salientam-se, a título de exemplo, a melhoria progressiva do tratamento da tuberculose pulmonar (DUARTE et al., 2010) bem como, a performance no acompanhamento e resposta à pandemia da gripe A e a diminuição do número de casos de infeção e da taxa de mortalidade por HIV/SIDA.

\section{Considerações finais}

A gestão das políticas de saúde em Portugal exige do assistente social um posicionamento analítico para que este possa entender os fenómenos onde é interventor. Assim, poderá ter a capacidade para produzir e construir conhecimento útil sobre a realidade social onde intervém, bem como para sugerir, implementar ou desenhar políticas alternativas com base na evidência da sua praxis. Algumas alterações decorrentes da interferência internacional no sistema de saúde português tiveram impactos significativos na intervenção dos profissionais de Serviço Social, a saber:

- ainda que não seja possível realizar ações padronizadas numa política que atua diretamente sobre a vida (IAMAMOTO, 2007) é imperativo identificar de forma transparente os processos de trabalho dos assistentes sociais, não como um fim em si, mas como um meio de proporcionar à população, a defesa dos seus direitos quando estes são colocados em causa;

- as necessidades das populações e a sua satisfação têm sofrido alterações decorrentes de um índice de longevidade crescente e do incremento da prevalência de doenças crónicas e incapacitantes - numa altura de racionalização do acesso à saúde e de não satisfação de necessidades por escassez de recursos na área da saúde. Alguns exemplos demonstrativos reveladores desta tendência estão relacionados, por exemplo, com o facto de muitos utilizadores dos serviços de saúde aguardarem demasiado tempo por cirurgias, consultas, meios complementares de diagnóstico e terapêutica e por outros tratamentos curativos/reabilitação;

- os assistentes sociais também lidam com alguns interesses instalados "na condução das políticas de saúde para o reforço das tendências de racionalizações das despesas públicas em saúde, da privatização dos seus serviços e da mercadorização das atividades dos seus profissionais" (CORTÊS; CARAPINHEIRO, 2013, p. 95) - comprometendo a essência da intervenção social. Por outras palavras, algumas estratégias de controlo de execução, a intensificação de processos ou procedimentos da ação (burocratização) acabam por deixar para segundo plano a reflexão do exercício profissional que lhe confere a sua natureza humanista e centrada no cidadão e nos seus sistemas de ação (VISCARRET, 2007);

- ao nível profissional, fustigados com a ausência de uma cultura assente na meritocracia, pela falta de perspetiva de progressão de carreira e pela pressão do cumprimento de objetivos e de resultados em saúde - os assistentes sociais lidam com dificuldades que foram, em parte, provocadas por um contexto de crise;

- porém, há outras contrariedades que se colocam às novas políticas de saúde. Estas relacionam-se com o afastamento do Estado e do setor público e com a responsabilização crescente da sociedade civil e da instituição familiar - dando espaço ao terceiro setor e ao setor privado um papel cada vez mais relevante no sistema de proteção social, longe da inspiração da criação do SNS que se revê na universalização e na gratuitidade dos serviços de saúde (BEHRING, 2012). Estes são alguns dos desafios que a categoria profissional se confrontou e se confronta no serviço nacional de saúde em Portugal.

\section{Referências}

ANTONOVSKY, A. The sctructural sources of salutogenic strengths. In: COOPER, C. L; PAYNE, R. (Eds.). Individual differences: Personality and stress. New York: Wiley, 1991. p. 67-104.

BECKET, C. Essential theory for Social Practice. London: Sage, 2009.

BEHRING, E. R. O estado social europeu tem futuro? In: VARELA, R. (Coord.). Quem paga o estado social em Portugal? Lisboa: Bertrand, 2012. p. 153-172.

CAMPOS, G. W. Saúde Paideia. São Paulo: Hucitec, 2003.

CAMPOS, A. C.; SIMÕES, J. O Percurso da Saúde: Portugal na Europa. Coimbra: Almedina, 2011.

CARMO, I. et al. Obesidade em Portugal e no mundo. Lisboa: Faculdade de Medicina da Universidade de Lisboa, 2008.

CORTÊS, S.; CARAPINHEIRO, G. Reformas de Sistemas de Saúde em Contextos de Reformas do Estado: Os Casos de Brasil e Portugal. In: ALVES, F. Saúde, Medicina e Sociedade: Uma Visão Sociológica. Lisboa: Pactor, 2013. p.75-98.

CRAIG, S., BEJAN, R.; MUSKAT, B. Making the Invisible visible: Are Health Social Workers Addressing the Social Determinants of health? Social Work in Health Care, n. 52, p. 311-331, 2013.

DAHLGREN, G.; WHITEHEAD, M. European strategies for tackling social inequities in health: Levelling up (part 2). Copenhagen: WHO Collaboration Centre for policy Research in Social Determinants of Health; Liverpool: University of Liverpool, 2006. 
DELOITTE PORTUGAL. Saúde em Análise: Uma visão para o futuro. Public Sector \& Healthcare. 2011. Deloitte. Disponível em: <http://www2.deloitte.com/content/dam/Deloitte/pt/Documents/life-sciences-health-care/pt(pt)_lshc_saudeemanalise_04022011.pdf.> Acesso em: 2 jun. 2016.

DIREÇÃO GERAL DA SAÙDE. Manual de Boas práticas para Assistentes Sociais na Saúde na Rede Nacional de Cuidados Continuados Integrados. Divisão da Qualidade. Lisboa: Direção de Serviços de Prestação de Cuidados de Saúde, Ministério da Saúde. 2007. Infotabac Relatório 2008-2010. Lisboa: Direção Geral de Saúde, Ministério da Saúde. 2011.

DUARTE, R. et al. Abordagem terapêutica da tuberculose e resolução de alguns problemas associados à medicação. Rev. Port. Pneumol., Lisboa, v. 16, n. 4, p. 559-572, ago. 2010. Disponível em: <http://www.scielo.mec.pt/scielo.php?script=sci_arttext\&pid=S0873$21592010000400004 \& \operatorname{lng}=$ pt\&nrm=iso $>$. Acesso em: 28 out. 2016.

EVERS, A.; WINTERSBERGER, H. (Eds.). Shifts in the welfare mix: Main impact on work, social services and welfare policies. California: Westview Press, 1990.

FALAGAS, M. E. et al. Economic crises and mortality: a review of the literature. International Journal of Clinical Practice, 63, p. 1128-1135, jul. 2009. Disponível em: <http://onlinelibrary.wiley.com/doi/10.1111/j.1742-1241.2009.02124.x/abstract>. Acesso em 27 out. 2016.

IAMAMOTO, M. Serviço Social em tempo de capital fetiche: capital financeiro, trabalho e questão social. São Paulo: Cortez, 2007. IFE, J. Human rights and social work: Towards rights-based practice, 3.ed. Cambridge, New York: Cambridge University, 2012. INTERNATIONAL FEDERATION OF SOCIAL WORKERS. INTERNATIONAL ASSOCIATION OF SCHOOLS OF SOCIAL WORK. INTERNATIONAL COUNCIL ON SOCIAL WELFARE. Global agenda for social workers and social development: commitment to action. 2012. Disponível em: 〈http://cdn.ifsw.org/assets/globalagenda2012.pdf>. Accesso em: 9 jun. 2016.

KARBAN, K. Social Work and Global health inequalities: Practice and policy developments. Social Work Education, v. 29, n. 8, p. 928 936, 2010

LUNDY, C. Social work, social justice \& human rights: a structural approach to practice. North York: University of Toronto Press, 2011. MARMOT, M. G.; BELL, R. How will the financial crisis affect health? British Medical Journal, 338, b.1314, abr. 2009. Disponível em: <https://doi.org/10.1136/bmj.b1314>. Acesso em 27 out. 2016.

MARTINS, A. Génese, Emergência e Institucionalização do Serviço Social Português: A Escola Normal de Coimbra. Revista Intervenção Social, Lisboa, n. 11/12, p. 17-34, dez. 1995.

MOZZICAFREDDO, J. Estado-Providência e Cidadania em Portugal. 2. ed. Oeiras: Celta Editora, 2000.

OBSERVATÓRIO PORTUGUÊS DOS SISTEMAS DE SAÚDE - OPSS. Relatório da Primavera de 2009: 10/30 Anos: razões para continuar. Coimbra: Mar da Palavra, 2009.

Relatório da Primavera de 2010: Desafios em tempos de crise. Coimbra: Mar da Palavra, 2010.

Relatório da Primavera de 2011. Da depressão da crise para a governação prospectiva da saúde. Coimbra: Mar da Palavra, 2011.

. Relatório da Primavera de 2014: Saúde. Síndroma de negação. Lisboa: Observatório Português dos Sistemas de Saúde, 2014.

PALMA, R. M. Prescrição de antibióticos no serviço de atendimento complementar. Revista Portuguesa de Clínica Geral, Lisboa, n. 18, p. 35-52, 2002.

PORTUGAL. Decreto-lei n 56/79, de 15 de Setembro de 1979. Criação do Serviço Nacional de Saúde. Diário da República nº 214/1979

- Série I. Assembleia da República. Lisboa. Disponível em: <https://dre.pt/>. Acesso em: 12 set. 2016.

Decreto-lei n. $^{\circ}$ 124/2011, de 29 de Dezembro de 2011. Linhas gerais do Plano de Redução e Melhoria da Administração Central. Diário da República no 249/2011 - Série I. Ministério da Saúde. Lisboa. Disponível em: <https://dre.pt/>. Acesso em: 12 jul. 2016.

Lei no 2011/46, de 2 de Abril de 1946. Lei da organização hospitalar. Diário do Governo, nº 70. Série I. Presidência da República. Lisboa. Disponível em: <https://dre.pt/>. Acesso em: 5 jun. 2016

Lei no27/2002, de 8 de Novembro de 2002. Aprova o novo regime jurídico da gestão hospitalar. Diário da República nº 258/2002

- Série I-A. Assembleia da República. Lisboa. Disponível em: 〈https://dre.pt/>. Acesso em: 16 jun. 2016.

MINISTÉRIO DAS FINANÇAS. Orçamento Estado. Relatório. 2012. Disponível em: <http://static.publico.pt/docs/economia/ PropRelOE2012.pdf>. Acesso em: 5 jun. 2016.

RESTREPO, O. Reconfigurando el Trabajo Social: perspectivas y tendencias contemporáneas. Buenos Aires: Espacio Editorial, 2003. SAKELLARIDES, C. Novo Contrato Social da Saúde - Incluir as Pessoas. Lisboa: Diário de Bordo, 2010.

SILVA, M. V. Políticas públicas de saúde: tendências recentes. Sociologia, Problemas e Práticas, Lisboa, p.121-128, mai./ago. 2012. UNITED NATIONS. Universal Declaration of Human Rights. 1948. Disponível em: <www.un.org/en/documents/udhr/index.shtml〉. Acesso em: 5 jun. 2016.

VISCARRET, J. J. Modelos y métodos de intervención en Trabajo Social. Madrid: Alianza, 2007.

\section{Jorge Lopes da Costa}

jlopesdacosta@hotmail.com

Assistente Social no Centro Hospitalar Lisboa Norte

Doutorado em Serviço Social pelo Instituto Superior de Ciências Sociais e Políticas (ISCSP) da Universidade de Lisboa 


\section{Maria Irene Carvalho}

mcarvalho@iscsp.ulisboa.pt

Doutorado em Serviço Social pelo Instituto Superior de Ciências Sociais e Políticas (ISCSP) da Universidade de Lisboa

Professora no Instituto Superior de Ciências Sociais e Políticas (ISCSP) da Universidade de Lisboa

\section{Carla Pinto}

cpinto@iscsp.ulisboa.pt

Doutorado em Política Social pelo Instituto Superior de Ciências Sociais e Políticas (ISCSP) da Universidade de Lisboa

Professora Auxiliar no Instituto Superior de Ciências Sociais e Políticas (ISCSP) da Universidade de Lisboa

\section{ISCSP - Universidade de Lisboa}

Rua Almerindo Lessa - 1300 - 663

Pólo Universitário do Alto da Ajuda

Lisboa - Portugal 